\title{
Programa de Cálculo para la Evaluación de Contaminantes en Efluentes Gaseosos y Pulverulentos de Hornos de Fundición
}

\author{
Gloria del V. Villaflor, Jorge W. Velasco y Eduardo R. Bisonard \\ Instituto de Beneficio de Minerales (INBEMI), Facultad de Ingeniería, Consejo de Investigaciones, \\ Universidad Nacional de Salta. Av. Bolivia 5150, 4400 Salta-Argentina \\ (e-mail: villaflo@unsa.edu.ar; inbemi@unsa.edu.ar)
}

Recibido Nov. 25, 2009; Aceptado Feb. 02, 2010; Versión Final recibida Abr. 20, 2010

\begin{abstract}
Resumen
Se presenta un programa de cálculo para evaluar la contaminación ambiental producida por efluentes gaseosos y polvos provenientes de hornos de fundición de procesos pirometalúrgicos. El sistema experimental consta de campanas para la captación de los efluentes gaseosos y polvos, equipos para decantación de partículas gruesas, enfriamiento de los gases y limpieza de polvos, conductos de aspiración y descarga, ventilador y chimenea. Se estiman las temperaturas a lo largo del sistema y se corrigen las alturas de velocidad y las pérdidas de carga. El programa efectúa los balances de energía del proceso, calcula el área del enfriador, las pérdidas de carga y la potencia del ventilador centrífugo. Permite además realizar cálculos de verificación, simular situaciones extremas y obtener resultados que ahorran tiempo de cálculo para una toma de decisión acertada, tanto para el funcionamiento correcto del sistema, como para establecer acciones conducentes a disminuir la contaminación producida por procesos pirometalúrgicos.
\end{abstract}

Palabras clave: efluentes gaseosos, polvos, contaminación ambiental, hornos de fusión, programa de cálculo

\section{Calculation Program for Evaluating Pollutants in Powdery and Gaseous Effluents from Melting Furnaces}

\begin{abstract}
The development of a software package for evaluating the environmental pollution due to gaseous effluents and powders from pirometallurgical processes furnaces is presented. The system consists of hoods to draw gaseous effluents and powders, decantation equipments for coarse particles, gas cooling and powder cleaning, aspiration and unloading ducts, fan and stack. The temperatures are estimated throughout the system, the heights speed and the load losses are corrected. The software performs the energy balances of the process, calculates the area of the cooler, the load losses and the centrifugal fan power. It also allows checking the calculations, simulates extreme situations and obtaining results that save calculation time for a successful decision making both for the proper function of the system and to establish the correct actions for reducing pollution produced by pirometallurgical processes.
\end{abstract}

Keywords: gaseous effluents, powders, environmental pollution, melting furnaces, calculation program 


\section{INTRODUCCIÓN}

Los procesos térmicos llevados a cabo en equipos tales como hornos, evaporadores, secaderos y calderas, que involucran procesos de combustión, transferencia de calor, transformaciones térmicas o termoquímicas de la carga tratada producen efluentes gaseosos con mayor o menor contenido de contaminantes. Estos efluentes pueden ser productos de la combustión (gases, material particulado) o producidos por reacciones químicas, reacciones pirometalúrgicas o decrepitación térmica, en forma de gases o polvos contaminantes. Para resolver estos problemas de importancia industrial es esencial la aplicación de modelos computacionales de transferencia de calor y flujo (Patankar, 2002). De los aportes de la literatura en dispositivos o métodos de captación, remoción o deposición de partículas, se mencionan: Hankinson (2005) que analiza el uso de campanas con cubiertas mecánicas en sistemas integrados de ventilación para evitar pérdidas de energía y disminuir los costos operativos. Chaichanawong et al. (2005) investigan nuevas técnicas de ionización de gases y partículas utilizando generadores de electrones de baja energía para producir iones negativos y partículas cargadas que luego son removidas. Wallin et al (1995) realizan un análisis de la importancia relativa de los distintos modelos de cálculo de la deposición de partículas en conductos y su comparación con datos experimentales de la literatura. Peters y Leith (2004) desarrollaron un método de medición de deposición de partículas en conductos utilizando rejillas de alambres lubricadas para captar las partículas. Asimismo Cascetta y Bellia (1996) comparan las fórmulas recomendadas con los datos experimentales, basado en la velocidad en las proximidades de la boca de entrada de las campanas de ventilación localizada. A fin de evaluar la contaminación ambiental de procesos industriales se hace necesaria la utilización de programas de simulación (Pérez et al, 2004). El objetivo principal de este trabajo es desarrollar una herramienta de cálculo que permita evaluar la contaminación ambiental producida por efluentes gaseosos y polvos provenientes de hornos de fusión de procesos pirometalúrgicos de metales como el plomo y el zinc, y determinar las acciones conducentes a disminuir dicha contaminación ambiental. Esta reducción debe hacerse protegiendo tanto el ambiente de trabajo como el ambiente exterior a la planta industrial. El objetivo específico es lograr el diseño preliminar del sistema de tratamiento de efluentes que minimice la contaminación ambiental según normas de la higiene industrial.

\section{DESARROLLO}

El horno es la fuente de emisión de los efluentes gaseosos, gases de combustión, material particulado y polvos o humos metalúrgicos. Se realizan cálculos de combustión para conocer la cantidad de aire necesario, el caudal de gases de combustión producidos, la composición y la temperatura de los mismos.

El sistema de ventilación consta de las siguientes etapas: captación de los gases provenientes del horno a través de campanas de diseño especial; decantación de partículas sólidas gruesas en cámaras de sedimentación y/o ciclones; enfriamiento de los gases efluentes a altas temperaturas; equipo de limpieza de polvos y partículas finas (ciclones, filtros de mangas); ventilador y sistema de evacuación de gases al exterior.

El sistema de captación de gases efluentes debe proporcionar un buen cerramiento para evitar corrientes transversales que desvíen el flujo de gases contaminantes (ACGIH, 1995), dependiendo de esto el caudal a aspirar, Q, por el sistema de ventilación. El caudal aspirado generalmente puede transportar partículas fundidas que solidifican en los conductos de aspiración, siendo recomendable colocar una cámara de sedimentación inmediatamente a la salida del horno.

La velocidad de diseño de los conductos de aspiración, $v_{d}$, permite calcular el diámetro de los conductos, D, para un caudal constante de gases a evacuar (Pocoví et al, 2002). Puede ser adoptada a partir de datos de la bibliografía o calculada con la velocidad mínima de transporte y un factor de seguridad $F_{\mathrm{s}}$ que tiene en cuenta factores tales como la humedad de las partículas y efectos electrostáticos que pueden aglomerar las mismas. La velocidad mínima de transporte depende fundamentalmente de la densidad y del tamaño de las partículas.

$\mathrm{v}_{\mathrm{d}}=\mathrm{F}_{\mathrm{s}} \mathrm{v}_{\min }$ 
$v_{\min }=5,2 \rho_{s}^{0,37} d_{p}^{0,26}$

$A=Q / v_{d} ; A=\pi D^{2} / 4 \rightarrow D$

El sistema de enfriamiento de los gases efluentes se selecciona de acuerdo a los requerimientos particulares del proceso, temperatura máxima soportada por las telas del filtro, cantidad de carga y humedad de los gases. De acuerdo a estos factores se puede seleccionar, por ejemplo, enfriadores de tubos con aire (circulación natural o forzada), con tolva para la separación de las partículas sólidas que decantan debido a cambios de dirección del flujo en los tubos.

Para el enfriamiento de fluidos en tubos en condiciones de flujo completamente turbulento $(\operatorname{Re}>$ 10000), con diferencias de temperaturas moderadas y con las propiedades (densidad $\rho$, viscosidad $\mu$ y conductividad térmica $k$ ) medidas en el seno del fluido, el coeficiente pelicular interno de transferencia de calor, $h_{i}$, se calcula como (Levenspiel, 1999):

$\frac{\mathrm{h}_{\mathrm{i}} \mathrm{D}}{\mathrm{k}}=0,023\left[1+\left(\frac{\mathrm{D}}{\mathrm{L}}\right)^{0,7}\right]\left(\frac{\mathrm{D} \mathrm{u} \rho}{\mu}\right)^{1 / 3}\left(\frac{\mu}{\mu_{\mathrm{w}}}\right)^{0,14}$

Para el enfriamiento por convección natural de cilindros verticales, de longitud $L>1 \mathrm{~m}$, se pueden usar ecuaciones simplificadas para calcular el coeficiente pelicular externo de transferencia de calor con aire en condiciones ambientales. Para fluidos que circulan sobre superficies en régimen laminar, turbulento y convección forzada, con velocidad del aire $u_{a}$, respectivamente, es:

$\mathrm{h}_{\mathrm{e}}=1,4\left(\frac{\Delta \mathrm{T}}{\mathrm{D}}\right)^{1 / 4}\left|\mathrm{~W} / \mathrm{m}^{2} \mathrm{~K}\right|$

$\mathrm{h}_{\mathrm{e}}=1,3(\Delta \mathrm{T})^{1 / 3}\left|\mathrm{~W} / \mathrm{m}^{2} \mathrm{~K}\right|$

$h_{e f}=h_{e}\left(1+0,69\left(u_{a}-0,75\right)\right)$

El coeficiente global $U$, despreciando la resistencia del tubo metálico, se calcula como:

$\frac{1}{\mathrm{U}}=\frac{1}{\mathrm{~h}_{\mathrm{i}}}+\frac{1}{\mathrm{~h}_{\mathrm{e}}}$

La cantidad de calor, $Q$, a extraer en el enfriador, siendo $T_{s}$ la temperatura de salida, menor a la máxima soportada por la tela del filtro es:

$Q=W_{g} \int_{T_{e}}^{T_{s}} c p_{g}(T) d T$

El área total de transferencia, $A$, requerida por el enfriador, con $\overline{\Delta T}_{\text {In }}$ calculada con las temperaturas del fluido caliente y la temperatura del ambiente exterior, es:

$\mathrm{A}=\frac{\mathrm{Q}}{\mathrm{U} \overline{\Delta \mathrm{T}}_{\mathrm{In}}}$

Como equipos de limpieza de los gases se pueden utilizar cámaras de sedimentación, ciclones, filtros de mangas y separadores electrostáticos. Para su selección se tiene en cuenta la concentración y tamaño de partículas del contaminante, eficiencia de separación (Pocoví et al, 2002), propiedades de la corriente gaseosa y del polvo y forma de descarga del polvo. 
Los filtros de tela dan una alta eficiencia de separación para polvos finos con carga de polvo en el gas baja. La temperatura máxima a la que pueden usarse está limitada por la resistencia de la tela filtrante, siendo de $80{ }^{\circ} \mathrm{C}$ aproximadamente para telas de algodón y de $280{ }^{\circ} \mathrm{C}$ para fibra de vidrio. Cuando el gas contiene vapor de agua, se debe mantener la temperatura de 20 a $30{ }^{\circ} \mathrm{C}$ por encima del punto de rocío.

El conducto de descarga consta de chimenea, ensamble ventilador-chimenea y dispositivo protector de lluvia. El cálculo de la chimenea se realiza teniendo en cuenta la velocidad con la cual deben salir los gases para dispersarse totalmente sin posibilidad de reingreso a la planta industrial.

El movimiento de los gases requerido en los sistemas de ventilación localizada se logra con ventiladores centrífugos, cuya potencia depende del caudal de aire a aspirar y de las pérdidas de presión que tienen lugar en los conductos de aspiración y descarga. Esto implica seleccionar correctamente el tipo de campanas de aspiración a utilizar y el tamaño de los conductos del sistema a fin de minimizar el consumo energético en el ventilador (Bhattacharjee, 2009).

\section{PROGRAMA DE CÁLCULO}

El programa para el cálculo del sistema de captación de contaminantes del horno de fusión determina la pérdida de carga de las líneas de aspiración y descarga, desde la campana de aspiración hasta la brida de succión del ventilador pasando por el enfriador y equipos de limpieza de polvos y desde la brida de descarga del ventilador hasta la salida de la chimenea, lo que permite obtener la potencia del ventilador a utilizar. La pérdida de carga en la campana $\Delta \mathrm{H}_{\mathrm{ph}}$ y en los conductos de aspiración $\mathrm{H}_{F}$, se calcula con la altura de velocidad $\mathrm{H}_{v}$ a la temperatura de los gases, $T$.

$$
\begin{array}{ll}
\mathrm{H}_{\mathrm{v}}=\frac{17,99}{\mathrm{~T}(\mathrm{~K})} \mathrm{v}^{2}(\mathrm{~m} / \mathrm{s}) & \left(\mathrm{mm} \mathrm{H}_{2} \mathrm{O}\right) \\
-\Delta \mathrm{H}_{\mathrm{ph}}=\mathrm{H}_{\mathrm{v}}\left(1+\mathrm{k}_{\mathrm{e}}\right) & \left(\mathrm{mm} \mathrm{H}_{2} \mathrm{O}\right) \\
\mathrm{H}_{\mathrm{F}}=\mathrm{n}_{\mathrm{v}} \mathrm{H}_{\mathrm{v}} \mathrm{L} \quad\left(\mathrm{mm} \mathrm{H}_{2} \mathrm{O}\right)
\end{array}
$$

$\mathrm{k}_{\mathrm{e}}$ es un factor que depende del ángulo de ahusamiento de la campana y $\mathrm{n}_{\mathrm{v}}$ es el número de velocidad calculado con el diámetro y la rugosidad del material del conducto, k (Pocoví, 1999):

$$
n_{v}=\frac{4 f}{D}=\frac{4}{D\left\{-4 \log \left[\frac{1}{3,7} \frac{k}{D}+\left(\frac{1,02}{10^{4} D v}\right)^{0,9}\right]\right\}^{2}}
$$

La pérdida de carga de los accesorios se calcula a partir de los factores de pérdida de carga, $\mathrm{K}_{\mathrm{v}}$. La

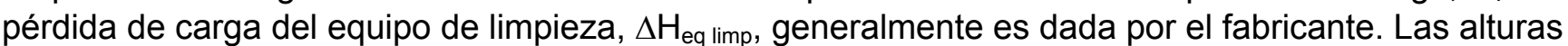
de presión en la brida de aspiración $\mathrm{H}_{\mathrm{ps}}$, de descarga $\mathrm{H}_{\mathrm{pd}}$ y altura total $\Delta \mathrm{H}_{\mathrm{T}}$, que permite calcular la potencia del ventilador a utilizar, se calculan con las siguientes expresiones (Villaflor, 2003):

$$
\begin{array}{ll}
\mathrm{H}_{\mathrm{p}_{\mathrm{s}}}=-\left(-\Delta \mathrm{H}_{\mathrm{ph}}+\mathrm{H}_{\mathrm{Fs}}+\sum \mathrm{H}_{\text {Facc }}+\Delta \mathrm{H}_{\text {eqlimp }}\right) & \left(\mathrm{mm} \mathrm{H}_{2} \mathrm{O}\right) \\
\mathrm{H}_{\mathrm{p}_{\mathrm{d}}}=\mathrm{H}_{\text {Fconex }}+\mathrm{n}_{\mathrm{vch}} \mathrm{H}_{\mathrm{v} \text { ch }} \mathrm{h}_{\mathrm{ch}} & \left(\mathrm{mm} \mathrm{H}_{2} \mathrm{O}\right) \\
\Delta \mathrm{H}_{\mathrm{T}}=\left(\mathrm{H}_{\mathrm{pd}}+\mathrm{H}_{\mathrm{vd}}\right)-\left(\mathrm{H}_{\mathrm{ps}}+\mathrm{H}_{\mathrm{vs}}\right) &
\end{array}
$$

Siendo $\mathrm{H}_{\mathrm{Fconex}}$ la pérdida de carga en la conexión ventilador-chimenea y $\mathrm{h}_{\mathrm{ch}}$ la altura de la chimenea. 


\section{RESULTADOS}

El programa, a través de hojas de cálculos específicos, permite definir la composición del combustible, el exceso y humedad del aire a utilizar y realizar los cálculos de combustión para conocer el caudal, la composición y la temperatura de los gases de combustión generados. Permite además calcular los coeficientes de transferencia de calor para convección natural y convección forzada y analizar la variación de los mismos y la influencia de la velocidad del aire proporcionada por ventiladores externos al enfriador, en el caso de convección forzada. Asimismo permite determinar las pérdidas de carga de las líneas de aspiración y descarga y la potencia del ventilador a utilizar. El usuario puede modificar datos de acuerdo a las necesidades impuestas por el proceso particular que quiere diseñar. A través de botones se pueden realizar saltos a otras hojas o pantallas que proveen información y resultados específicos correspondientes a las operaciones y equipos que componen el sistema de tratamiento estudiado. A modo de ejemplo se muestran los resultados del sistema ventilación realizado para un efluente gaseoso de dos hornos de fusión para tratar escorias de zinc y plomo, con gran producción de polvos contaminantes. El consumo de combustible en cada horno es de $100 \mathrm{~m}^{3} / \mathrm{h}$ de gas natural y el exceso de aire del $10 \%$. En la Figura 1 se muestra la pantalla de cálculo principal del programa con los caudales de aire utilizados y gases de combustión producidos y en la Figura 2 se muestran los cálculos de la pérdida de carga en los conductos y del ventilador centrífugo a utilizar.

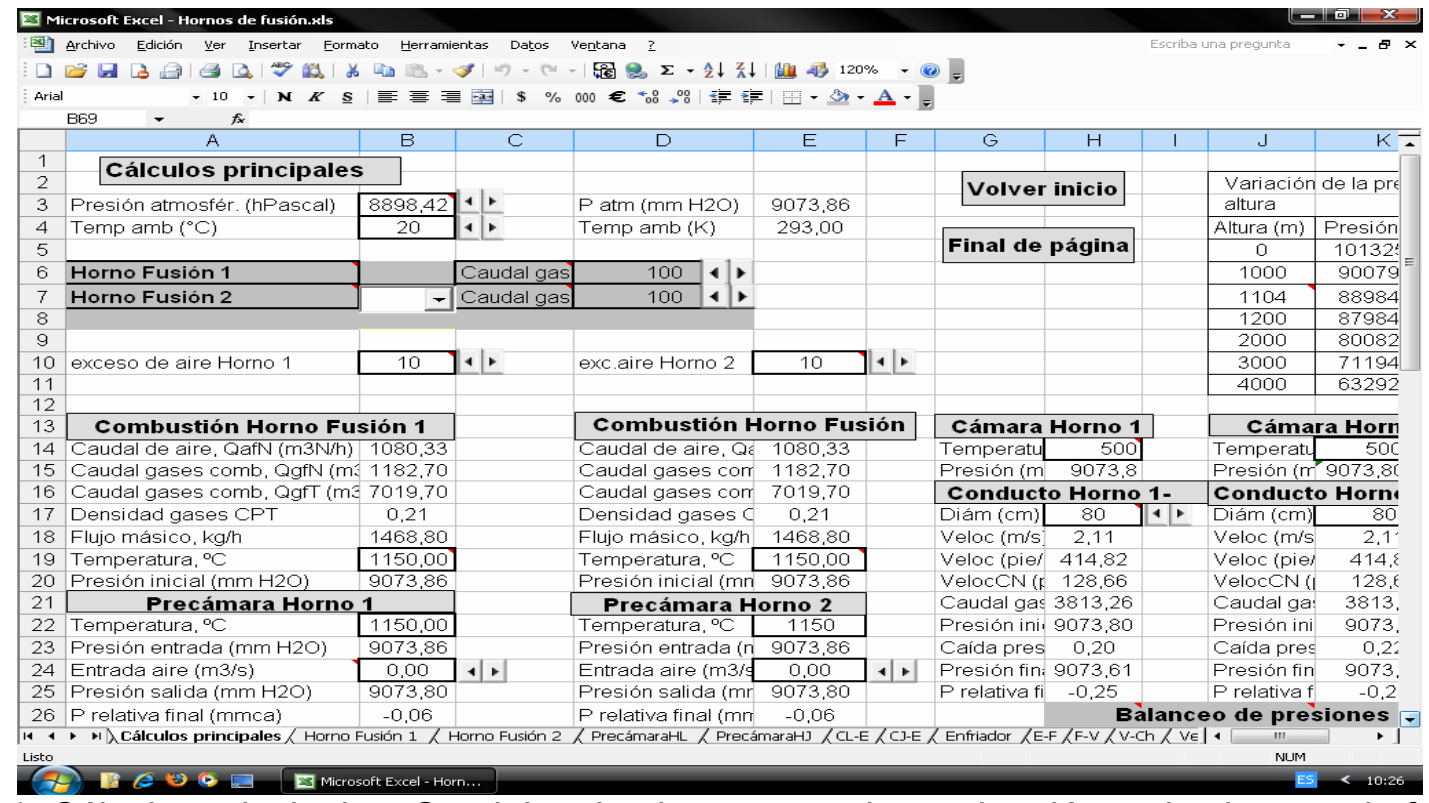

Fig. 1: Cálculos principales. Caudales de aire y gases de combustión en los hornos de fusión

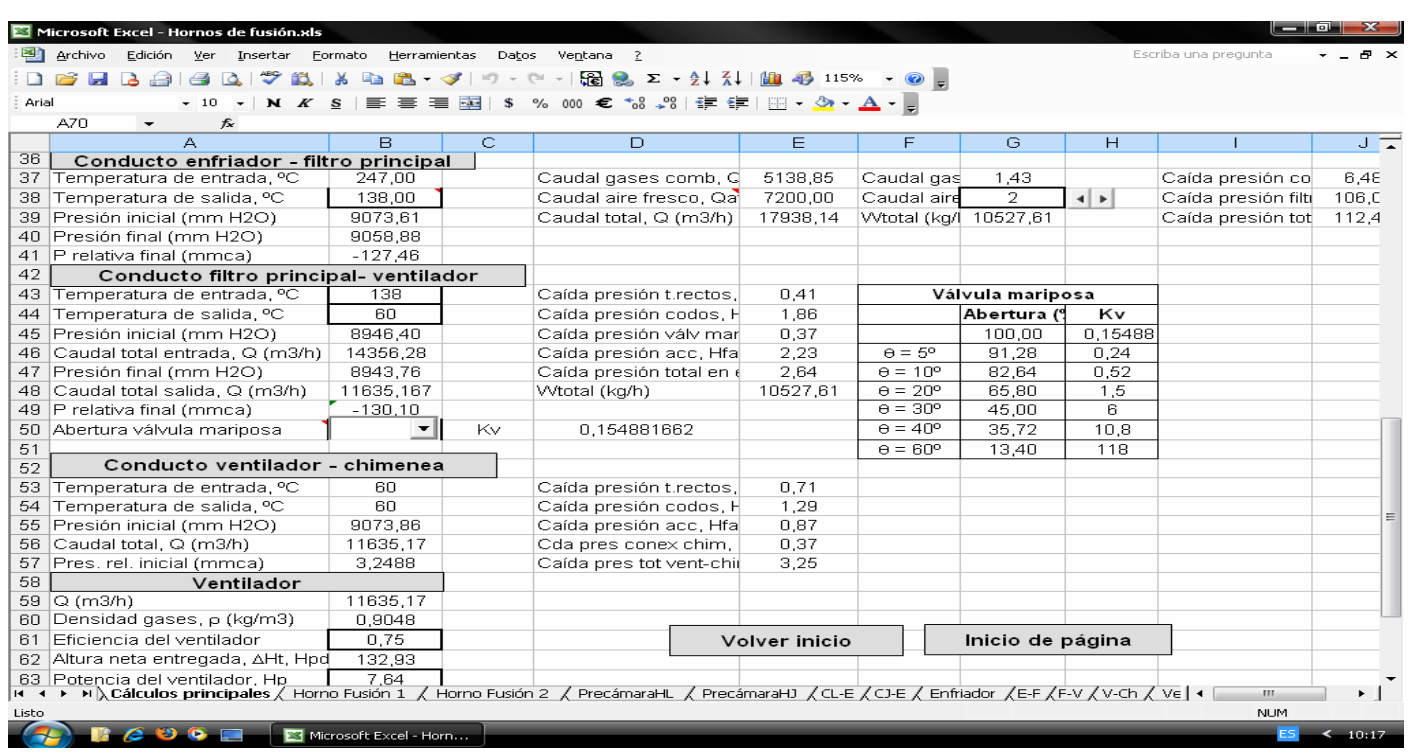

Fig. 2: Pérdidas de carga en conductos y cálculo del ventilador 


\section{CONCLUSIONES}

El programa de cálculo desarrollado permite: i) realizar la verificación de sistemas de ventilación de hornos de fusión; ii) simular situaciones extremas; iii) analizar y discutir los resultados para distintas condiciones operativas; iv) obtener o identificar los parámetros y las condiciones de trabajo adecuadas; v) tomar decisiones tanto para el funcionamiento correcto del sistema estudiado como para establecer acciones conducentes a disminuir la contaminación ambiental producida por procesos térmicos o pirometalúrgicos.

\section{REFERENCIAS}

American Conference of Governmental Industrial Hygienists (ACGIH), Industrial Ventilation A Manual of Recommended Practice, $22^{\text {nd }}$ Edition. ACGIH, USA (1995).

Bhattacharjee K.; Energy Conservation Opportunities in Industrial Ventilation Systems. Energy Engineering: 106(3), 73-79 (2009).

Cascetta, F. y L. Bellia; Velocity fields in proximity of local Exhaust hood openings: an intercomparison between current recommended formulas and experimental studies. Building and Environment: 31(5), 451-459 (1996).

Chaichanawong, J. y otros cinco autores; High-temperature simultaneous removal of acetaldehyde and ammonia gases using corona discharge. Science and Technology of Advanced Materials: 6, 319324 (2005).

Hankinson, K.; The Best-Kept Secret for Minimizing Your Energy Costs. Metal Finishing: 103(11), 1317 (2005).

Levenspiel, O.; Flujo de fluidos e intercambio de calor. Ed. Reverté, España (1993).

Patankar, S.; Computational modeling of flow and heat transfer in industrial applications. Internacional Journal of Heat and Fluid Flow: 23, 222-231 (2002).

Pérez S.A., Z. M. Niño y M.J. Llobregat; Desarrollo de un Programa de Simulación de Procesos para el Tratamiento de Emisiones Atmosféricas. Información Tecnológica: 15 (2), 55-62 (2004).

Peters T. y D. Leith; Measurement of particle deposition in industrial ducts. Journal of Aerosol Science: 35, 529-540 (2004).

Pocoví, R. y otros cinco autores; Diseño de criterios para instalaciones de ventilación localizada. Criterio para maximizar la eficiencia de separación. Revista Innovación: 1(14), 25-34 (2002).

Pocoví, R.; Ventilación Industrial. Descripción y diseño de los sistemas de ventilación industrial. Ed. Magna Publicaciones, Argentina (1999).

Villaflor, G. y otros tres autores; Programa para el diseño de sistemas complejos de ventilación localizada; Actas del VI CAIP: 383-386. (2003).

Wallin, O. y T. G. Malmstrom; Particle deposition models for ventilation air ducts. Int. J. HVAC\&R Res., ASHRAE: 1(3), 194-218 (1995). 